\title{
Numerical Simulations of the Transient Flow Response of a 3D, Low-Aspect-Ratio Wing to Pulsed Actuation
}

\author{
Guillaume A. Brès*, \\ Exa Corporation, Brisbane, CA 94005 \\ Ehab Fares ${ }^{\dagger}$ \\ Exa GmbH, 70563 Stuttgart, Germany \\ David R. Williams, \\ Illinois Institute of Technology, Chicago, IL 60616 \\ and \\ Tim Colonius ${ }^{\S}$ \\ California Institute of Technology, Pasadena, CA 91125
}

\begin{abstract}
Numerical simulations of the natural and actuated unsteady flow over a three-dimensional low-aspect ratio wing are performed using Lattice Boltzmann method. The LBM simulations match the flow conditions and the detailed wing geometry from previous experiments, including the actuators that are installed internally along the leading edge of the wing. The present study focuses on the transient lift response to short-duration square-wave actuation, for the wing in a uniform flow at five different angles of attack. Overall, both mean and unsteady numerical results show good agreement with the experimental data, in particular at the post-stall angle of attack $19^{\circ}$, where the maximum lift enhancement occurs. At that angle of attack, the effects of the actuation strength and duration are investigated. In general, the lift response to a single pulse increases with increasing actuator mass-flow rate and pulse duration.
\end{abstract}

\section{Nomenclature}

$\begin{array}{ll}C_{D} & \text { Drag coefficient } \\ C_{L} & \text { Lift coefficient } \\ C_{\mu} & \text { Momentum coefficient } \\ c & \text { Chord } \\ D & \text { Drag } \\ f & \text { Vortex shedding frequency } \\ h & \text { Airfoil thickness } \\ I_{t} & \text { Total lift impulse } \\ L & \text { Lift } \\ P & \text { Pressure } \\ Q & \text { Actuator mass flow rate } \\ R e & \text { Chord Reynolds number } \\ S & \text { Planform area } \\ S t & \text { Strouhal number }\end{array}$

$\begin{array}{ll}t & \text { Time } \\ U_{0} & \text { Freestream velocity } \\ \alpha & \text { Angle of attack } \\ \Delta D & \text { Transient change in drag } \\ \Delta L & \text { Transient change in lift } \\ \Delta t_{\text {on }} & \text { Pulse duration } \\ \nu & \text { Kinematic viscosity } \\ \text { Superscript } \\ +\quad \text { Quantity scaled with convective time } U_{0} / c \\ - & \text { Time averaged quantity } \\ \text { Subscript } \\ 0 \quad \text { Freestream quantity } \\ \text { jet } & \text { Quantity at the actuator jet exit }\end{array}$

*Present address: Cascade Technologies, Palo Alto CA; gbres@cascadetechnologies.com; Senior member AIAA

$\dagger$ Technical Manager, Aerospace Applications; Senior member AIAA

¥Professor, Dept. of Mechanical, Materials, and Aerospace Engineering; Associate Fellow AIAA

$\S$ Professor, Dept. of Mechanical Engineering; Associate Fellow AIAA 


\section{Introduction}

For wings at high angles of attack in unsteady flow conditions, the ability to increase lift or delay the onset of flow separation via active flow control is of interest. Such an ability is particularly relevant for micro-air vehicles (MAV) performing rapid maneuvers or responding to gusting flows. While flow control is well characterized for steady-state situations, ${ }^{1}$ its direct application to unsteady flow conditions has been limited. In addition, experimental data on the three-dimensional effects that dominate the flow physics for low-aspect ratio planforms is limited. Recently, several experimental investigations were conducted to improve the understanding of the unsteady aerodynamic flow physics ${ }^{2-5}$, including the three-dimensional effects. ${ }^{6-10}$ Colonius and Williams11 review these experiments in the context of controlling vortex shedding. In contrast, few numerical studies are available, and are often limited to two-dimensional geometry in steadystate flow conditions. ${ }^{12}$

In our previous work, ${ }^{13}$ numerical simulations of the natural and actuated unsteady flow over a semicircular planform wing were performed using a computational fluid dynamic (CFD) approach based on the Lattice Boltzmann method (LBM). The LBM simulations exactly matched the unsteady flow conditions and detailed three-dimensional geometry in the experiments, ${ }^{7-10}$ including the actuators that are installed internally along the leading edge of the wing. Overall, this first computational study showed good agreement with experiment and demonstrated the feasibility of numerical simulations of fully detailed MAV geometry.

As a next step towards a better understanding of unsteady flow control, the current paper focuses on the transient response of the separated flow to an individual actuator pulse. The motivation behind the choice of a single pulse of actuation is to examine the impulse response of the flow, which would ultimately be used as part of more complex prediction models. Details on the experimental configuration and numerical setup are presented in section II. The simulations are performed at five different angles of attack and the results with the flow control both on and off are compared with experiments ${ }^{7}$ in section III. For angle of attack $19^{\circ}$, the effects of the actuation strength and duration are investigated and discussed in section IV.

\section{Experimental and computational setup}

\section{A. Experimental procedure}

The test model used in this study is a low-aspect-ratio, low Reynolds number semi-circular planform wing mounted on a vertical sting, ${ }^{8,9}$ as shown in figure 1 . The wing centerline chord (i.e., radius), span and thickness are $c=0.203 \mathrm{~m}, b=2 c$, and $h=0.069 c$, respectively. The wing aspect ratio is defined as $A R=b^{2} / S=2.54$, where $S=1 / 2 \pi c^{2}$ is the planform area. The freestream velocity is $U_{0}=5 \mathrm{~m} / \mathrm{s}$, resulting in a chord Reynolds number $R e=U_{0} c / \nu \approx 68000$.

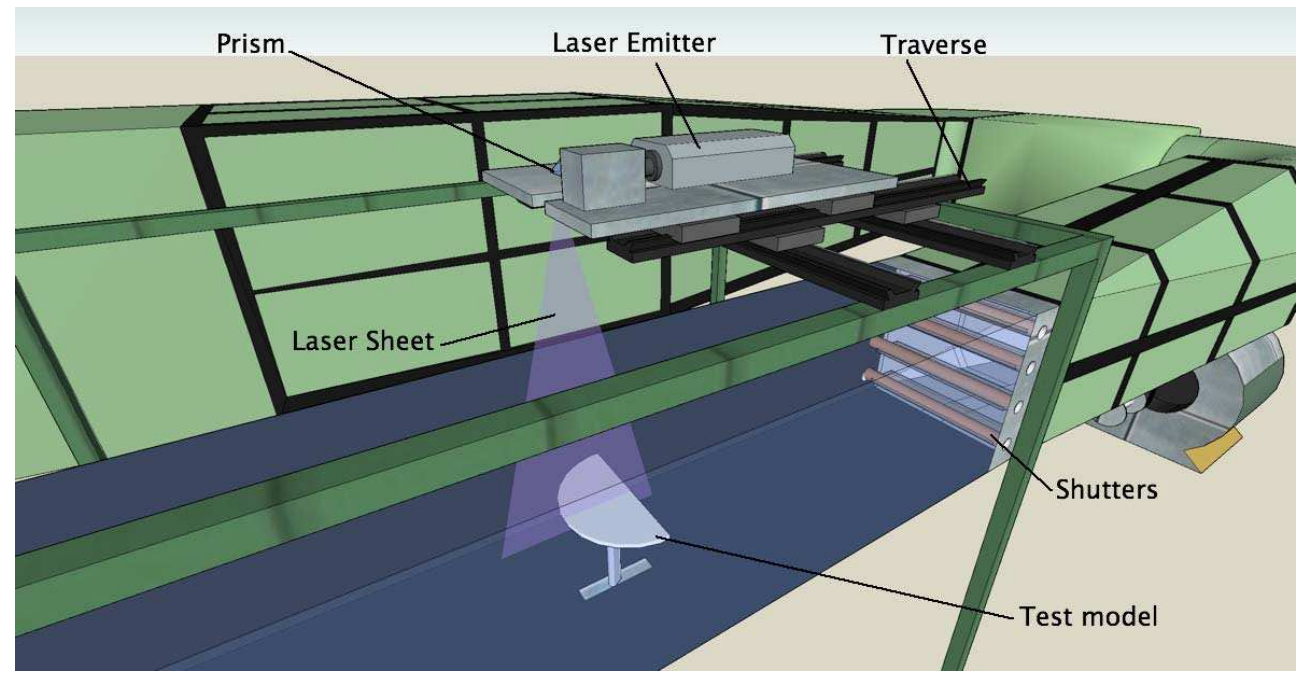

Figure 1. Schematic of the Andrew Fejer Unsteady Wind Tunnel at IIT. Flow is from left to right. The test model is the semi-circular planform wing in the center of the test section. 
For the active flow control, sixteen micro-valves are installed internally along the leading edge of the wing. All the actuators are driven in phase. The present study focuses on short-duration square-wave actuation, to reproduce recent experimental results where the lift increment due to a single actuator pulse was studied. ${ }^{7}$ In the experiment, the micro-valves are open for a duration $\Delta t_{o n}=0.017 \mathrm{~s}$, with a constant supply pressure. Here, the time of the single pulse was chosen to be small compared with the response times of the flow. The lift and drag forces acting on the semi-circular wing are recorded, with and without leading edge actuation, for angles of attack, $\alpha=0^{\circ}$ to $30^{\circ}$. The uncertainty in the force measurement is based on the repeatability of the calibration data and can be estimated to approximately $0.05 \mathrm{~N}$. There is more uncertainty in the measurement of mass flow rates, in particular for the small values.

\section{B. Numerical setup}

The CFD code PowerFLOW 4.2b based on the Lattice Boltzmann method ${ }^{14-17}$ is used in this study. Coupled with turbulence modeling, ${ }^{18,19}$ the LBM scheme has been well validated in recent years ${ }^{20-22}$ and used extensively for problems involving unsteady flows over complex geometries. ${ }^{23-26}$

The CAD geometry of the experimental wing was used to generated the computational mesh. Most of the internal piping for the actuators and the wing support are removed in the simulations, as shown in figure $2(a)$, and $(b)$. However, the detailed geometry at the leading edge of the wing (i.e., the rim and the end of each actuator pipe) is resolved in order to capture the actuator flow physics. In the simulations, the pulsed-blowing actuation is imposed as a mass-flow boundary condition on the back wall inside of each actuator pipe, as indicated by the red arrow in figure $2(b)$.

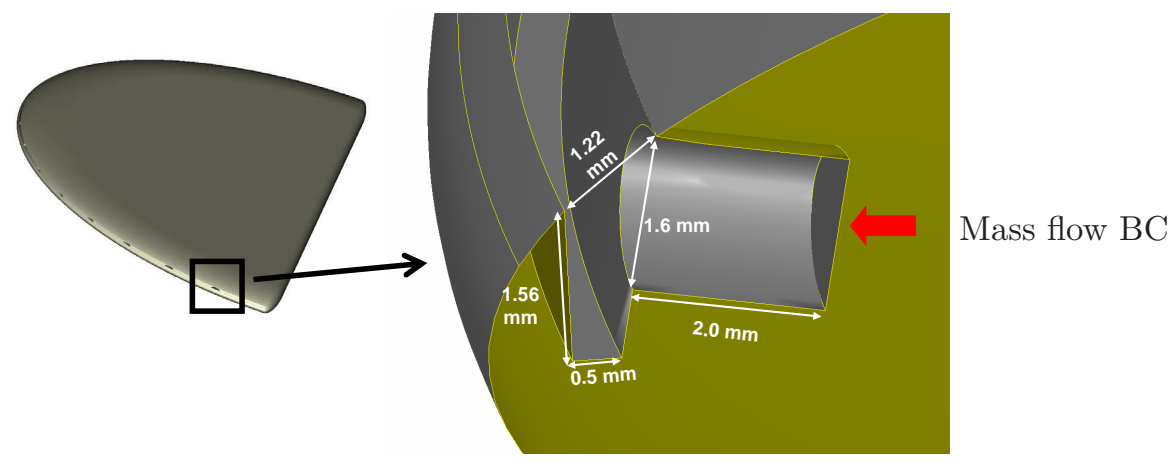

(a) Wing model

(b) Zoomed view of one actuator

Figure 2. Schematic of the wing geometry for the LBM simulation. The red arrow indicates the mass flow boundary condition (BC) used in the simulation to reproduce the experimental actuation.

The numerical scheme is solved on a grid composed of cubic volumetric elements, with embedded variable resolution regions to allow for successive refinement of the grid structure in regions of high gradients in the flow or where geometric details need to be resolved. Based on previous resolution studies, ${ }^{13}$ the resolution is concentrated near the wing, with a cell size of $0.4 \mathrm{~mm}$ at the surface, corresponding to approximately 500 cells per chord length. To properly capture the effects of the actuators, an additional level of grid refinement is added locally inside the rim and actuator pipe, and around the wing leading edge. The finest cell size is 0.2 $\mathrm{mm}$, and the total number of cells is about 57 millions. It is important to note that we do not fully resolve the boundary layer inside the actuators, which would be expensive and without much expected benefit. For the same reason, the wall boundary conditions in the rim and actuators are set to frictionless.

In the numerical study, five angles of attack are considered: $\alpha=0^{\circ}, 10^{\circ}, 16^{\circ}, 19^{\circ}$ and $30^{\circ}$. The other simulation parameters match the experimental flow condition, including the freestream velocity $U_{0}=5 \mathrm{~m} / \mathrm{s}$ and the Reynolds number $R e=68000$.

For each angle of attack, a coarse computation is first performed with the wing initially in a uniform flow $U_{0}$, and zero flow inside the rim and the actuator pipes, to speed up temporal convergence. The baseline and actuated simulations are then initialized from the flow field of the coarse computation, using a seeding technique. The short initial transient is discarded and the total simulated time is about $1 \mathrm{~s}$. The total computational cost of one simulation is approximately $4500 \mathrm{CPU}$ hours, which is about one and a half day on 128 processors. 
For the actuated cases, the mass flow boundary condition is turned on after the initial transient for a duration $\Delta t_{\text {on }}=0.017 \mathrm{~s}$ (i.e., duration normalized by convective time $\left.\Delta t_{\text {on }}^{+}=\Delta t_{\text {on }} U_{0} / c=0.42\right)$ with a constant total mass flow rate $Q=1.8810^{-3} \mathrm{~kg} / \mathrm{s}$ (summed for all sixteen actuators), unless stated otherwise. These conditions correspond to the experiments ${ }^{7}$ with actuator supply pressure of $34.5 \mathrm{kPa}(5 \mathrm{psi})$.

\section{Natural and actuated flows}

\section{A. Baseline cases}

After the initial transient, the time averaged forces are computed for the baseline simulations (i.e., no actuation). The forces are nondimensionalized by $1 / 2 \rho_{0} U_{0}^{2} S$, and the lift and drag coefficients are reported as a function of the angle of attack in figure $3(a)$ and $(b)$, respectively. In these figures, the experimental lift and drag forces are measured using an ATI force 6-component balance system, during a slow pitch motion of the wing, at 0.5 degrees per second pitch rate. ${ }^{10}$ Overall, agreement within the uncertainty of the experimental data is obtained at all five angles of attack, for both the lift and drag forces. The wing stalls around $\alpha \approx 14^{\circ}$ in the experiment, and a similar result is obtain in the simulation.

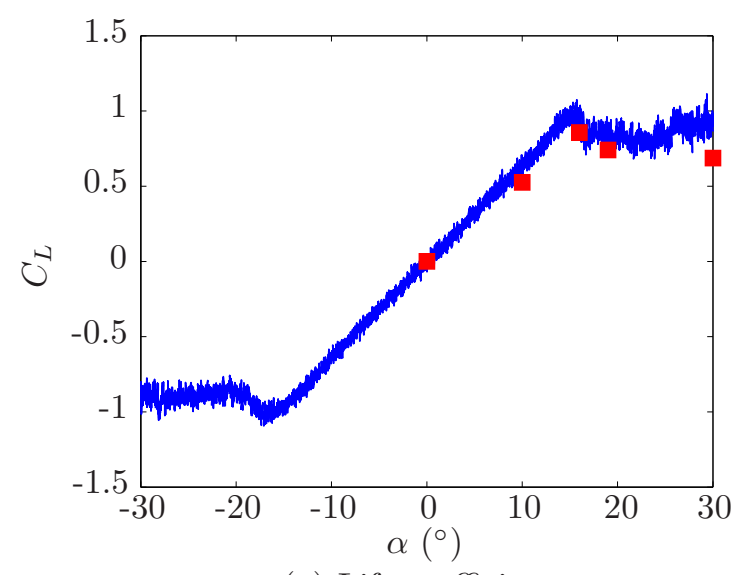

(a) Lift coefficient

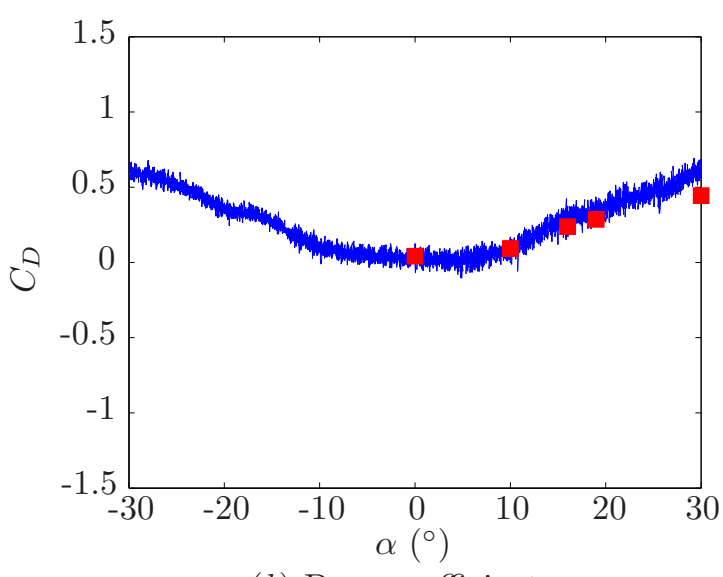

(b) Drag coefficient

Figure 3. Steady force coefficients on the wing without flow actuation, as a function of the angle of attack $\alpha$, from experiments ( - ) and LBM simulations ( $\square$ )

The instantaneous vorticity magnitude in the mid-span plane is shown in figure 4. For pre-stall angles of attack (e.g., $\alpha=10^{\circ}$ ), the boundary layer remains attached, and strong vortex shedding is observed starting at the blunt trailing edge. As the angle of attack is increased (e.g., $\alpha=16^{\circ}$ ), separation occurs at the leading edge and intermittent reattachment around the mid-chord can be observed. At $\alpha=19^{\circ}$, the flow over the wing is fully detached. The separated shear layer is apparently Kelvin-Helmholtz unstable, and rolls up into fine vortical structures which are subsequently merged into a larger, lower frequency structure near the trailing edge which is reminiscent of bluff-body vortex shedding.

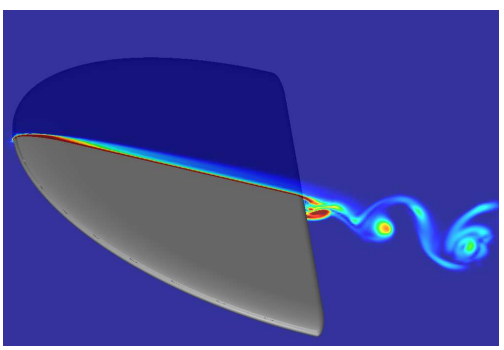

(a) $\alpha=10^{\circ}$

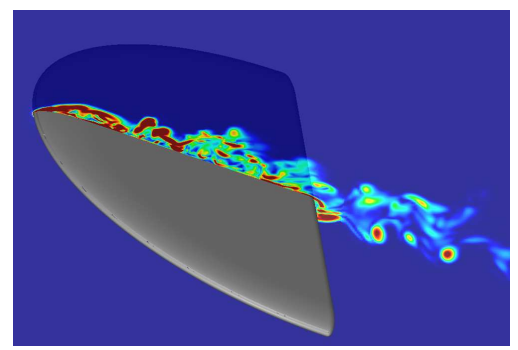

(b) $\alpha=16^{\circ}$

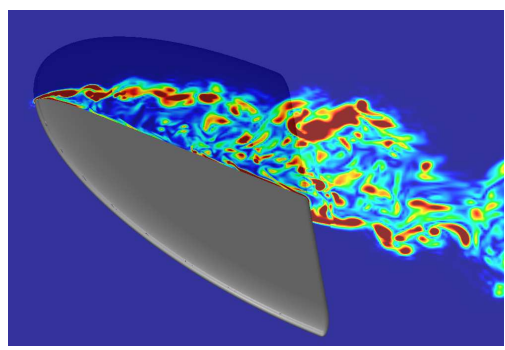

(c) $\alpha=19^{\circ}$

Figure 4. Instantaneous vorticity magnitude in the mid-span plane from the LBM simulation without actuation. 


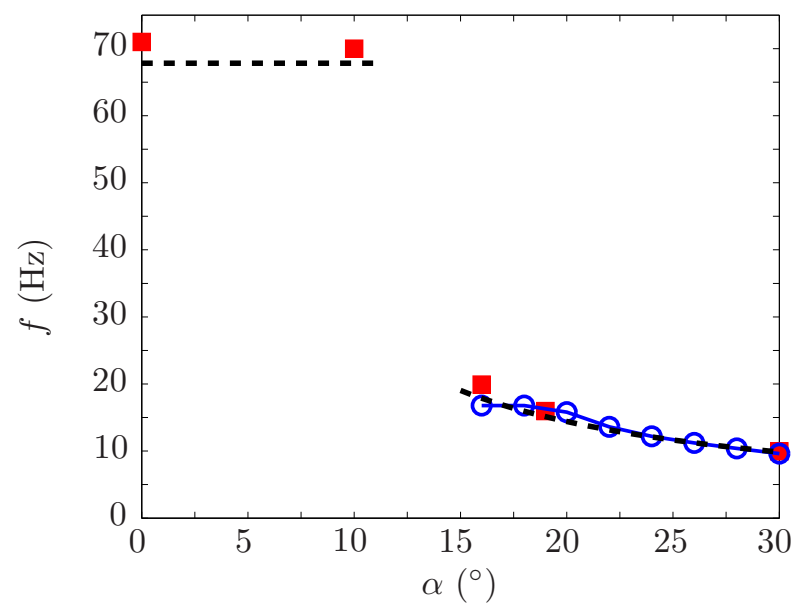

Figure 5. Vortex shedding frequency without flow actuation, as a function of the angle of attack $\alpha$, from experiments ( $\bigcirc-)$, LBM simulations ( - ), and theory ( ---- ).

A vortex shedding frequency, $f$, is estimated from the spectra of the lift and drag forces at the different angles of attack. The numerical results are compared to experimental measurements and theory in figure 5 . Here, the theoretical vortex shedding frequency is based on bluff-body type shedding and computed using a Strouhal scaling $S t=f c \sin (\alpha) / U_{0} \approx 0.2$. Overall, the comparison show good agreement above $\alpha=15^{\circ}$, when the flow over the wing is fully separated. Below that angle of attack, there is no dominant peak in the experimental spectra. For the simulations at angle of attack $\alpha=0^{\circ}$ and $10^{\circ}$, the flow is still attached and the vortex shedding occurs at the trailing edge of the wing. In these cases, the dominant frequency in the force spectra is $f \approx 70 \mathrm{~Hz}$. Applying the simple physical arguments suggested by Roshko and Bearman, ${ }^{27}$ the mechanism of the vortex shedding could be connected to the two shear layers at the top and bottom surfaces of the wing, separated by a distance $h$ (i.e., the wing thickness), with outside velocity $U_{0}$. A wake Strouhal number can then be formed, $S t=f h / U_{0} \approx 0.19$. This leads to a theoretical frequency of approximately 68 $\mathrm{Hz}$ similar to the numerical result. Additional analysis, for probes at various location in the shear layer and wake, is underway to confirm these results and report on the evident high and low frequencies in the flow field.

\section{B. Characteristics of the pulsed actuation}

Figure 6 shows the time history (in convective units $t^{+}=t U_{0} / c$ ) of the velocity measured at the actuator exit. In the simulation, the flow properties are recorded for each actuator approximately at the center of the pipe cross-section just before the rim, and averaged over all the actuators. Based on the pipe dimensions and the specified mass flow rate, the expected exit velocity is about $12.5 \mathrm{~m} / \mathrm{s}$, which matches the maximum measured velocity $U_{j e t}$ right after the actuation start. However, because of the presence of the rim, the average velocity over the pulse duration is slightly lower, $\bar{U}_{\text {jet }} \approx 10.9 \mathrm{~m} / \mathrm{s}$. For the present geometry, the near-actuator flow field is complicated and a single point measurement cannot be expected to fully characterize it. Future simulations will include additional measurements near the pipe exit and the rim to improve the characterization of the pulsed actuation.

Overall, there is reasonable agreement between simulation and experiment for the jet exit velocity. The maximum value in the initial peak is slightly higher and the mean velocity response is typically lower in the measurements. Here, because the distance between the two hotwire probe tips are larger than the width of the exit section, it is not possible to locate the hotwire exactly at the center of (or inside) the actuator pipe. Also, it is clear that the experiment has different actuator dynamics, due to the fact that there is piping between the valve and the outlet. Further analysis is needed to determine whether the oscillations in the measurements correspond to the acoustic impedance of the piping.

The momentum coefficient $C_{\mu}=\rho U_{a c t}^{2} S_{a c t} /\left(0.5 \rho_{0} U_{0}^{2} S\right)$ is typically used to characterize the amplitude of the actuation, where $U_{a c t}$ and $S_{a c t}$ are the characteristic velocity and area of actuation, respectively. Different values can be obtained for $C_{\mu}$ depending on whether the mean or fluctuating actuation velocity 


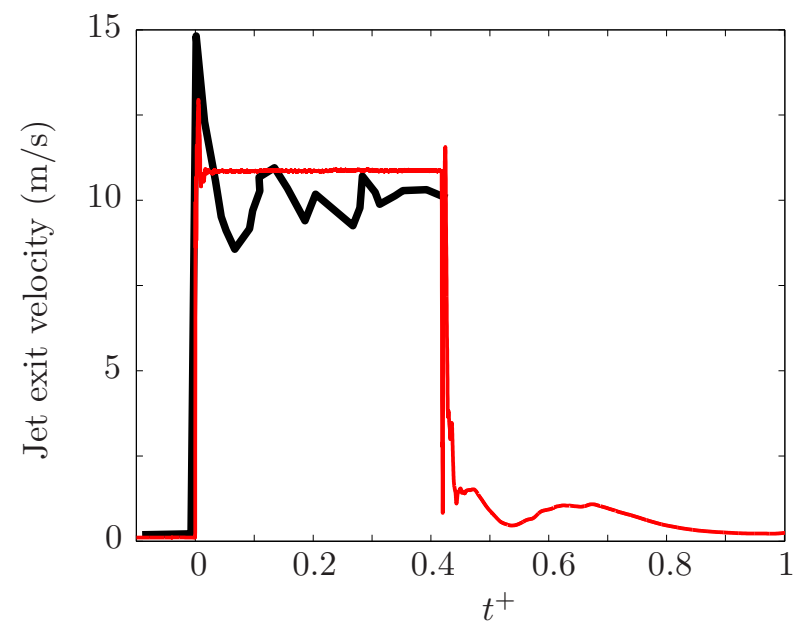

Figure 6. Time history of the jet exit velocity for the pulsed actuation case, from experimental hotwire measurement ( $\longrightarrow$ ), and from the LBM simulations ( $(-)$ ).

is used as characteristic velocity. For $U_{a c t}=\bar{U}_{\text {jet }}$ and $U_{a c t}=U_{\text {jet }}$, the mean and fluctuating values of the momentum coefficient are $\bar{C}_{\mu} \approx 0.018$ and $C_{\mu} \approx 0.026$, respectively. In both cases, the momentum coefficient is small but values as low as about $10^{-4}$ can be effective. ${ }^{28}$

\section{Actuated cases}

The comparison of the transient forces on the wing with and without actuation is presented in figure 7 , for angle of attack $\alpha=16^{\circ}, 19^{\circ}$, and $30^{\circ}$. The start and end of the pulsed square wave actuation can clearly be observed in the time history of lift and drag in figure 7(a). For the three angles of attack, the flow control leads to small changes in drag.

Similar to what has been observed in experiments, ${ }^{10}$ the single pulse actuation has little effect on the lift and drag for low angles of attack, when the boundary layer is attached. In the simulations at $\alpha=0^{\circ}$ and $10^{\circ}$, both transient and mean force coefficients change by less than $1 \%$, with and without control. ${ }^{13}$ This trend is also observed here for $\alpha=16^{\circ}$, even though the flow experiences intermittent separation and reattachment. As the angle of attack is increased and the flow becomes fully separated, the effect of the pulse actuation is significant. In the experiments, lift enhancement occurred over the range of angles from $16^{\circ}$ to $30^{\circ}$, with a maximum around $\alpha=20^{\circ}$. The simulations show a similar behavior.

The transient change in lift $\Delta L$ between the actuated and baseline cases is computed as a function of time, and compared to the experiment in figure $7(b)$. In this figure, the times are shifted to align the start of the pulsed square wave actuation at $t^{+}=0$, and the red line corresponds to the experimental valve voltage, representative of the pulse duration. It is important to note that the experimental $\Delta L$ is the phase-averaged lift response to a single pulse of the actuator relative to the mean baseline lift. As described in Ref. 7, the phase-averaged signal is constructed by averaging 59 cycles, with the pulse start as phase reference. The 59 cycles of data are obtained in a continuous run with a $5 \mathrm{~s}$ delay after each pulse to allow the flow sufficient time to reestablish equilibrium before the next pulse occurs. For the simulations, only one cycle of $1 \mathrm{~s}$ is available at this stage. As an attempt to approximate the phase average procedure, the numerical $\Delta L$ is computed as a function of time by taking the difference between lift from the actuated and baseline cases (i.e., difference between blue and black curve in figure $7(a)$ ). Additional simulations are underway to enable phase-averaging and validate the robustness for this approximation.

As mentioned in the previous paragraphs, the effectiveness of the actuation is limited at low and very high angles of attack. This trend is observed for both experiment and simulations at $\alpha=16^{\circ}$ and $30^{\circ}$ in figure $7(b)$. In the latter case, the dominant features are the lift fluctuations associated with the natural vortex shedding frequency discussed in section IIIA, rather than the a clear lift increment response to the pulse actuation.

For the angle of attack $\alpha=19^{\circ}$, the simulation captures the main features of the transient changes in lift. In the first stage after the actuation is initiated, a short transient with negative lift increment, known 

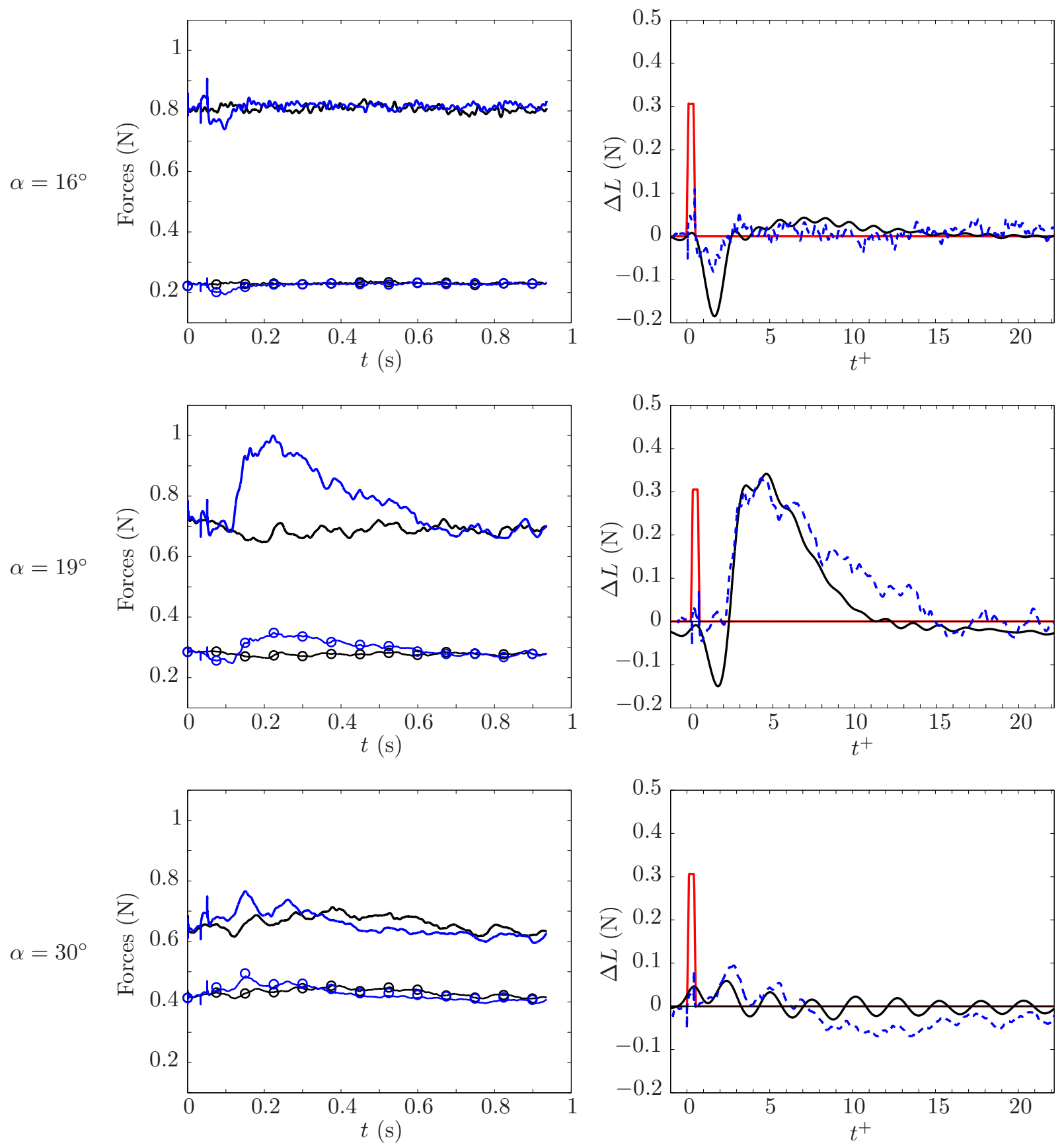

(a)

(b)

Figure 7. Transient forces on the wing at angle of attack $\alpha=16^{\circ}, 19^{\circ}$, and $30^{\circ}$ : (a) Time history of lift (solid line) and drag (circle line) from LBM simulations for baseline ( - ) and actuated cases ( - ); $(b)$ Transient change in lift $\Delta L$ from experiment ( - ) and LBM simulations ( ---- ). The red line indicates the duration of the square pulse actuation. 
as non-minimum phase behavior in the control community, is observed. Similar behavior has been reported in other previous experiments ${ }^{3,29}$ with pulse actuation. The initial decrease is present in the simulation but under predicted. In the next stage, the lift rises rapidly and increases until it reaches a maximum value around $t^{+} \approx 5$. The last stage corresponds to a slow relaxation from the maximum lift value back to the baseline flow state. While there is good agreement between the LBM results and experimental data for the time and value of the maximum lift, the total duration of the transient increase in lift is slightly over predicted in the simulation, $t^{+} \approx 15$, instead of $t^{+} \approx 11$ in the experiment. This over prediction of the predicted $\Delta L$ are likely due to the lack of phase-averaging in the computations. Nevertheless, these values are similar to $t^{+} \approx 10$ measured in other experiments ${ }^{5,30,31}$ with different actuators.

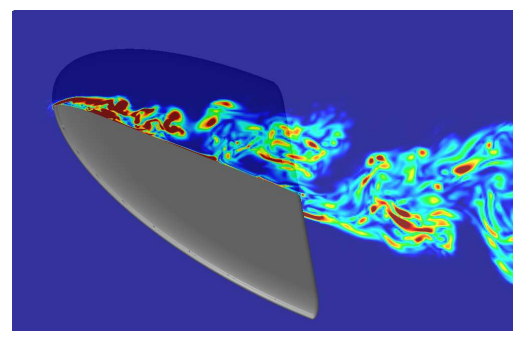

(a) $t^{+}=1.7$

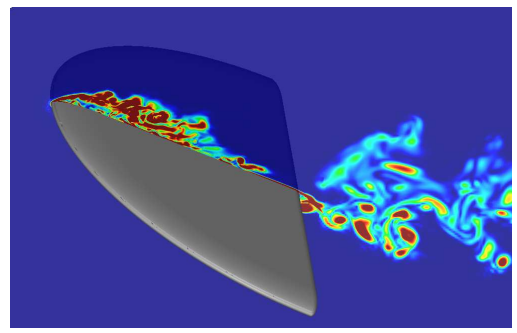

(b) $t^{+}=2.9$

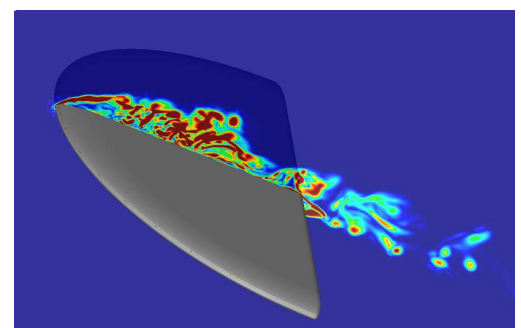

(c) $t^{+}=4.1$

Figure 8. Instantaneous vorticity magnitude at $\alpha=19^{\circ}$ from the LBM simulation with the experimental pulsed actuation, for the convective time $t^{+}$(measured from the start of the pulse) increasing from left to right.

To give some further insight on the mechanism behind the transient increase in lift, three snapshots of the vorticity field at $\alpha=19^{\circ}$ for the LBM simulation with actuation $\left(\Delta t_{o n}^{+}=0.42\right.$, mass flow rate $Q)$ are presented in figure 8 . This visualization matches the location (mid-span plane) and the vorticity magnitude levels (from 0 to 50) used for the natural flow presented in figure $4(c)$. The instantaneous flow field in figure $(a),(b)$, and $(c)$ correspond approximately to convective time $t^{+}=1.7,2.9$ and 4.2 after the start of the pulse actuation. The results reveal a process similar to that previously documented for a two-dimensional airfoil ${ }^{30}$ and observed for the current wing model using phase-locked PIV along the wing midspan. ${ }^{13}$ During and just after the pulse, a negative (counterclockwise) vortex is formed as the actuator jet pushes into the separated region, and begins a process where the entire separated region is detached and advected downstream. Following this, a fresh leading edge vortex (LEV) begins to form and is eventually shed, in a process that appears similar to that which occurs following the initial separation on an impulsively started aerofoil and the dynamic stall process during rapid pitch up.

\section{Parametric study of the transient flow response to pulsed actuation}

\section{A. Effect of the actuation strength}

To further investigate the flow response to actuation, simulations are performed with different mass flow rates, at angle of attack $\alpha=19^{\circ}$. The actuator mass flow rates considered are $6 \%, 12 \%, 25 \%, 50 \%, 75 \%$, and $100 \%$ of the experimental value $Q=1.8810^{-3} \mathrm{~kg} / \mathrm{s}$. Following the same procedure described in section IIIB, the maximum (i.e., initial transient) and mean (i.e., time-averaged over the pulse duration) jet exit velocity and pressure are measured for the different cases and presented in figure 9 . Note that the ambient pressure $P_{0}$ has been removed for the reported pressure.

As expected, the mean jet exit velocity scales linearly with the mass flow rate, and, in every case, is about $20 \%$ lower than the peak value because of the blockage effect of the rim. Likewise, the mean exit pressure follows the typical velocity square scaling. In contrast the maximum pressure grows linearly. This peak value is measured during the short transient spike right after the actuation start, similar to the spike observed in the velocity in figure 6. Additional analysis and experimental measurements are underway to investigate this feature.

The lift and drag increments are shown in figure 10(a) and $(b)$ respectively, for the different mass flow rate actuation. Qualitatively, the results show that the maximum value and total duration of the transient change in lift increase with mass flow rate to eventually saturate for mass flow rates greater than $50 \%$ of the 


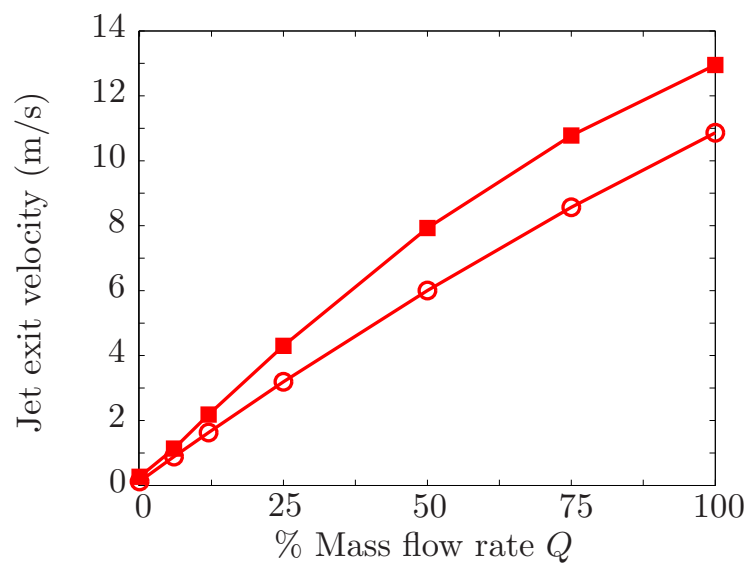

(a)

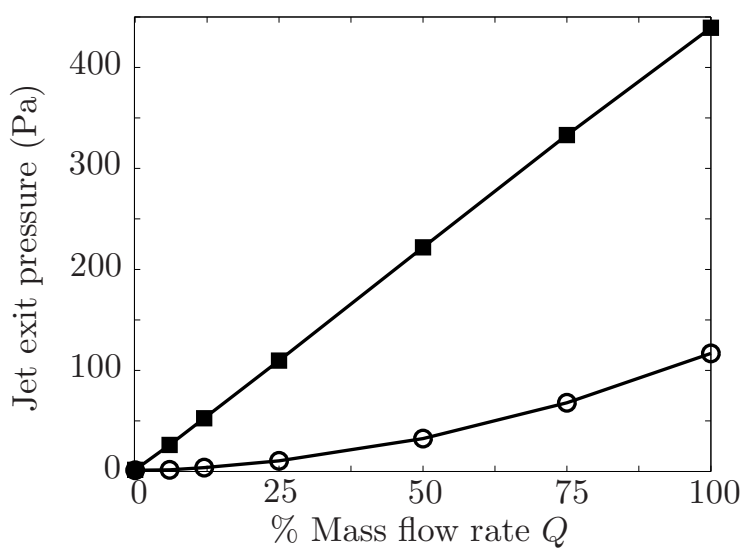

(b)

Figure 9. Jet exit velocity and pressure for the pulse actuation case with different mass flow rates, from the LBM simulations: (a) Maximum and mean jet exit velocity, $U_{\text {jet }}$ ( $\bullet$ ) and $\bar{U}_{\text {jet }}(\circ)$; (b) Maximum and mean jet exit pressure, $P_{j e t}(\boldsymbol{m})$ and $\bar{P}_{\text {jet }}(\circ)$

experimental actuation. The time when maximum lift occurs is largely independent of the actuation mass flow rate, and corresponds to $t^{+} \approx 4.2$. As before, the impact of the actuation on drag is limited and similar in all the cases.

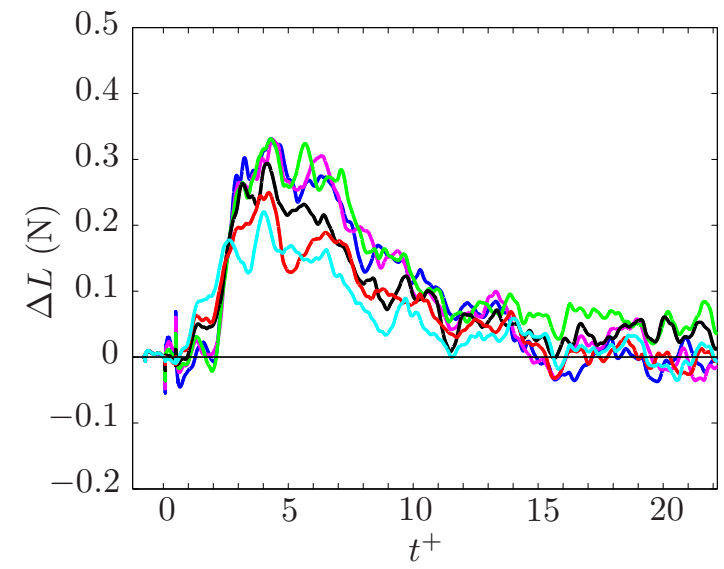

(a) Lift

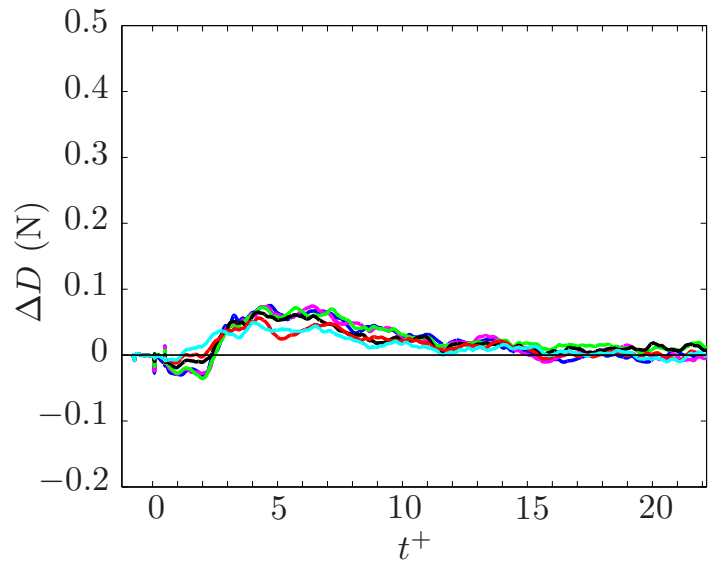

(b) Drag

Figure 10. Transient change in forces on the wing at $\alpha=19^{\circ}$ in the LBM simulations, for actuated cases of mass flow rate $6 \%(-), 12 \%(-), 25 \%(-50 \%(-), 75 \%(-)$, and $100 \%(-$ ( $(-)$ of the experimental mass flow rate $Q$.

For a more quantitative analysis of this data, the total lift impulse $I_{t}$ and the maximum lift increment $\max (\Delta L)$ are computed and reported in figure $11(a)$ and $(b)$, respectively. The lift impulse is the time integral of the transient increase in lift from the start of the actuation to the end of the simulation (i.e., the area under the lift curves in figure 10(a)). For a given flow speed, the lift curves scaled by the total impulse have been shown to collapse onto a single curve in the experiment. ${ }^{7}$ Similarly, reasonable collapse can be obtained with the present computations, but the lift impulse is challenging to compute reliably because of the short simulation time and the lack of phase averaging. Therefore, in figure 11( $a)$, the decrease in $I_{t}$ for the higher speeds, corresponding to $75 \%$ and $100 \%$ mass flow rate, is likely due in large part to the cycle-to-cycle variations in the lift response and the resulting time integration of the lift curve. Here, the maximum lift increment shows less sensitivity to the numerical procedure, leads to a similar collapse of the data and might be more reliable for the analysis of the numerical results. Nevertheless, both $I_{t}$ and $\max (\Delta L)$ highlight two different regimes for the lift response: a linear regime where the transient lift grows almost linearly with the 
actuation strength, up to $U_{\text {jet }} / U_{0} \approx 1.6$ (i.e., $50 \%$ of the experimental mass flow rate); then a saturation regime, where no further increase in lift is obtained with higher mass flow rate.

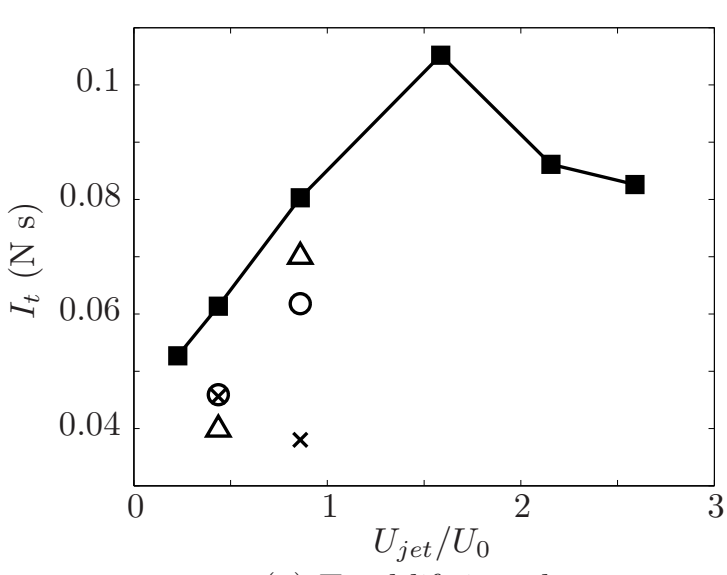

(a) Total lift impulse

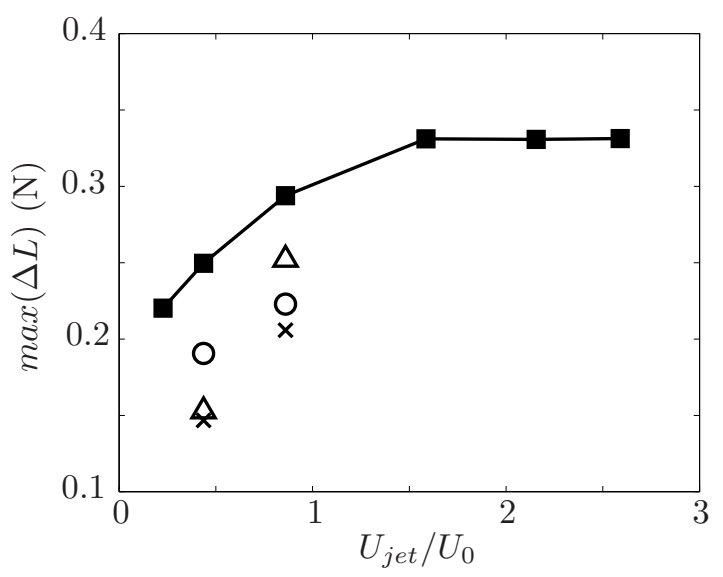

(b) Maximum lift increment

Figure 11. Total lift impulse and maximum lift increment at $\alpha=19^{\circ}$ in the LBM simulations as a function of the actuator exit velocity, for pulse duration $\Delta t_{o n}=0.017 \mathrm{~s}(\mathbf{\bullet}), 0.01 \mathrm{~s}(\triangle), 0.004 \mathrm{~s}(\circ)$, and $0.002 \mathrm{~s}(\times)$.

\section{B. Effect of the actuation duration}

The influence of the pulse duration on the lift is investigated in the simulations by decreasing the experimental value $\Delta t_{o n}=0.017 \mathrm{~s}$ to $0.01 \mathrm{~s}, 0.004 \mathrm{~s}$ and $0.002 \mathrm{~s}$ (i.e., $\Delta t_{o n}^{+}=0.42,0.25,0.1$ and 0.05 ). This study is done for $12 \%$ and $25 \%$ of the experimental mass flow rate, that is, in the linear growth regime and not the saturated state. These conditions would be challenging to reproduce in the experiments because of the low mass flow rate required and the limitations on the time response of the actuator valve.

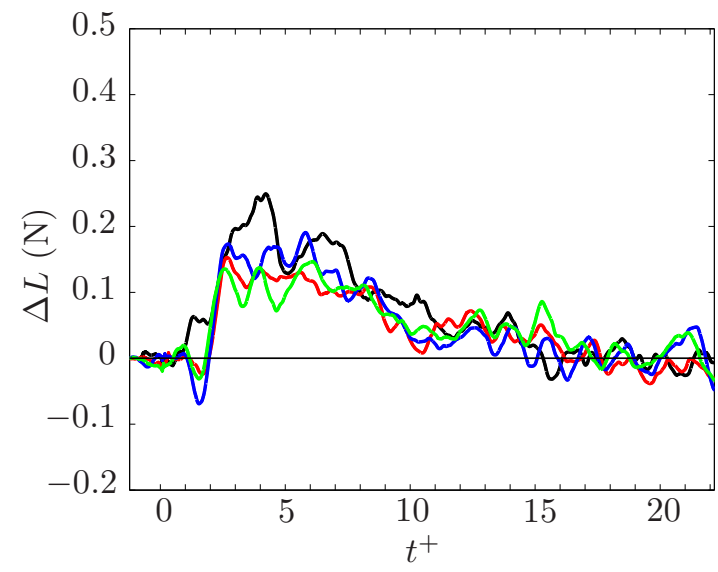

(a) $12 \% \mathrm{Q}$

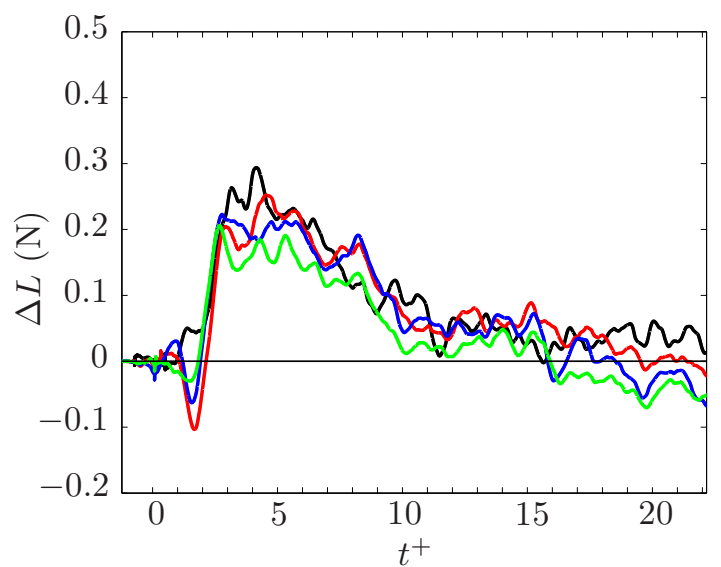

(b) $25 \% \mathrm{Q}$

Figure 12. Transient change in lift at $\alpha=19^{\circ}$ in the LBM simulations, for actuated cases of pulse duration $\Delta t_{\text {on }}=0.017 \mathrm{~s}(\longrightarrow), 0.01 \mathrm{~s}(-), 0.004 \mathrm{~s}(\longrightarrow)$ and $0.002 \mathrm{~s}(\longrightarrow)$, at $12 \%$ and $25 \%$ of the experimental mass flow rate $Q$.

The transient change in lift for $12 \% Q$ and $25 \% Q$ are presented in figure $12(a)$ and $(b)$ respectively. For the four different pulse durations, the increase in lift is qualitatively similar. For the case at $25 \%$ mass flow rate, the momentum coefficient is $C_{\mu} \approx 0.0029$ and is further reduced to 0.0007 at $12 \%$ mass flow rate. Here, the actuation seems to remains effective even for these small values of $C_{\mu}$, and even for pulse duration about one order of magnitude smaller than the experimental value.

Additionally, the short initial transient with negative lift increment discussed in section IIIC is more 
pronounced with the shorter pulses. The results also tend to indicate that the amplitude of this initial transient could depend mainly on pulse duration rather than amplitude.

The total lift impulse $I_{t}$ and the maximum lift increment $\max (\Delta L)$ are reported in figure 13 for the different pulse durations, as well as in figure 11 as a function of the actuation exit velocity. As expected, at a given mass flow rate, the maximum and mean flow properties at the actuator exit are independent of the pulse duration and $U_{\text {jet }} / U_{0}$ remains constant in these cases. Overall, $I_{t}$ and $\max (\Delta L)$ tend to increase with $\Delta t_{o n}^{+}$, although there is large uncertainty on the prediction at very low mass flow rate and very short pulse, in particular for the total lift impulse for the reasons discussed in the previous sections.

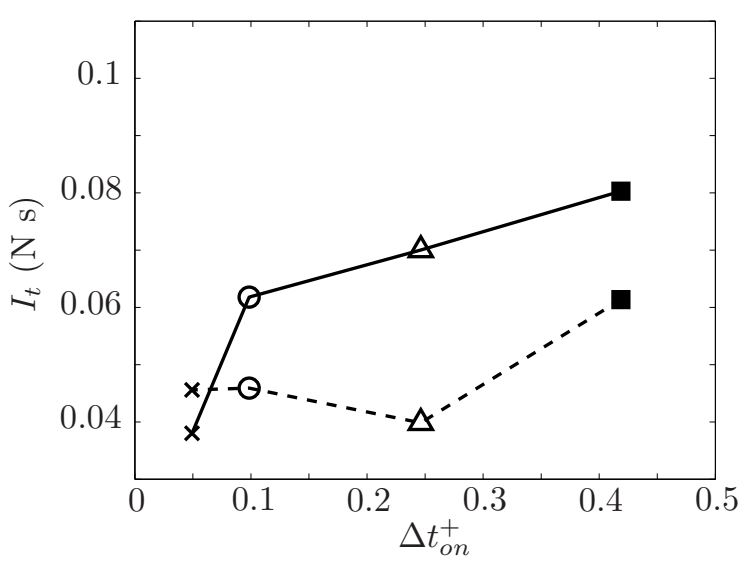

(a) Total lift impulse

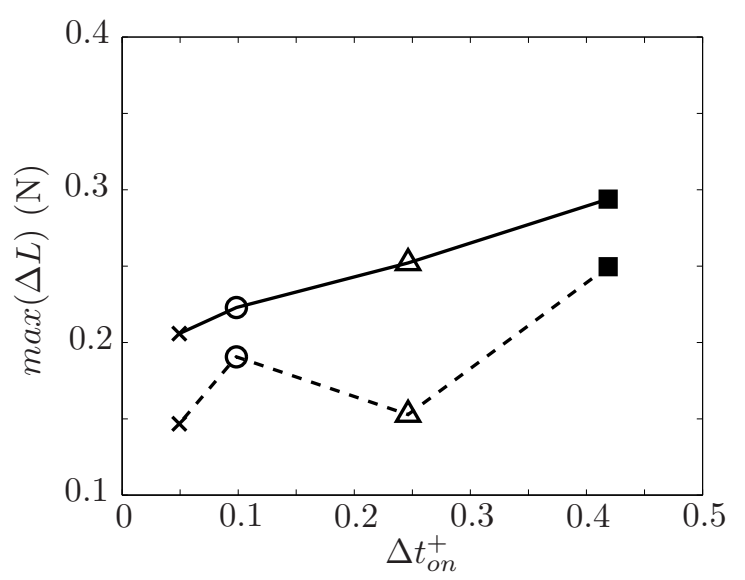

(b) Maximum lift increment

Figure 13. Total lift impulse and maximum lift increment at $\alpha=19^{\circ}$ in the LBM simulations as a function of the pulse duration, for $12 \%(----)$ and $25 \%$ ( -$)$ of the experimental mass flow rate.

For all the simulations at angle of attack $\alpha=19^{\circ}$, the maximum lift coefficient increment $\max \left(\Delta C_{L}\right)$ is computed and reported in figure 14 for comparison with the experimental data from Ref. 11 . The measurements are done at $\alpha=20^{\circ}$ for a wide range of freestream velocity $U_{0}=2.5$ to $8 \mathrm{~m} / \mathrm{s}$ and pulse duration $\Delta t_{o n}=0.005$ to $0.06 \mathrm{~s}$ ( or $0.06<\Delta t_{o n}^{+}<2$ ), and for the actuator supply pressure from 3.45 to $34.5 \mathrm{kPa}$. Overall, both experiment and simulation results show the same trends, in particular the nearly linear increase of lift increment with $U_{\text {jet }} / U_{0}$ up to about 1.6. There is however some scatter in both data sets, and an over prediction of the lift increase for the lower mass flow rate in the computations. These discrepancies are likely due to the uncertainties on the force measurements and the actuator exit velocity in the experiments, and to the lack of phase averaging in the simulations.

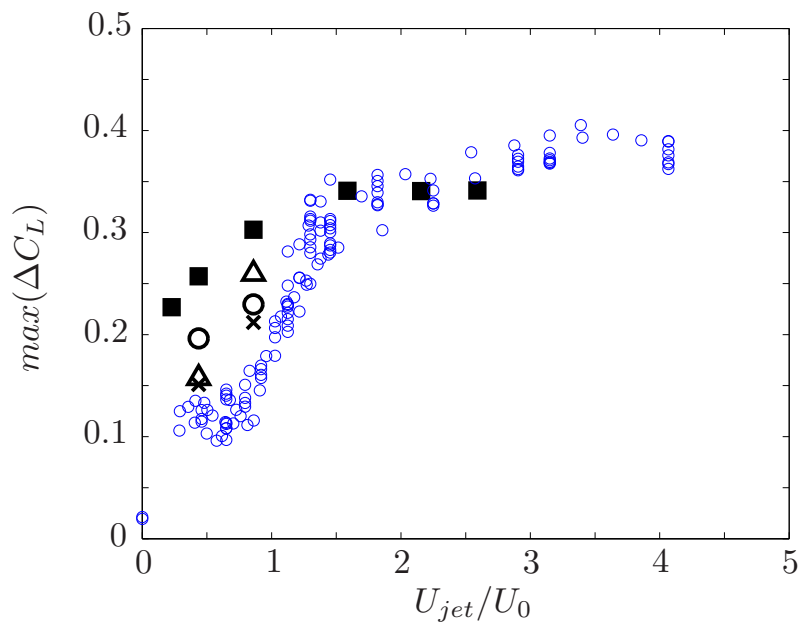

Figure 14. Maximum lift coefficient increment in experiment $(\circ)$, and in the LBM simulations for pulse duration $\Delta t_{o n}=0.017 \mathrm{~s}(\mathbf{a}), 0.01 \mathrm{~s}(\Delta), 0.004 \mathrm{~s}(\bigcirc)$, and $0.002 \mathrm{~s}(\times)$. 


\section{Conclusions}

Numerical simulations of the unsteady flow over a three-dimensional semi-circular planform wing are performed using Lattice Boltzmann method. The LBM simulations match the flow conditions (freestream velocity $U_{0}=5 \mathrm{~m} / \mathrm{s}$, chord Reynolds number $R e=68000$ ) and the detailed wing geometry in the experiment, including the actuators that are installed internally along the leading edge of the wing.

Five angles of attack $\alpha=0^{\circ}, 10^{\circ}, 16^{\circ}, 19^{\circ}$ and $30^{\circ}$ are investigated in the simulations, with flow control both on and off. To reproduce the single pulse actuation used in the experiment, a short-duration squarewave actuation is imposed in the simulations as a mass-flow boundary condition in the actuators. For both mean and unsteady lift and drag, the numerical simulations show good agreement with the measurements. In particular, the maximum lift increment occurs at the post-stall angle of attack $\alpha \approx 20^{\circ}$ in the experiment and the simulations capture a similar behavior.

For the angle of attack $\alpha=19^{\circ}$, the effects of the actuation strength and duration are investigated. Simulations are performed for lower mass flow rates and shorter pulses duration than in the experiment. In general, the lift response increases with increasing actuator mass-flow rate and pulse duration. There is however some uncertainty on the prediction at very low mass flow rate and very short pulse, mainly because of the limited simulation time. These issued will be addressed in future work with longer simulations and phase averaging to reduce the cycle-to-cycle variations in the lift response.

\section{Acknowledgments}

The authors would like to thank Vien Quach and Wesley Kerstens from IIT, for details of the measurement and additional experimental data. Caltech and IIT have been supported by the US AFOSR under grants FA9550-05-1-0369 and FA9550-09-1-0189.

\section{References}

${ }^{1}$ Seifert, A., Darabi, A., and Wygnanski, I., "Delay of Airfoil Stall by Periodic Excitation," J. Aircraft, Vol. 33, No. 4, 1996, pp. 691-698.

${ }^{2}$ Amitay, M. and Glezer, A., "Controlled Transients of Flow Reattachment over Stalled Airfoils," Int. J. Heat and Fluid Flow, Vol. 23, No. 5, 2002, pp. 690-699.

${ }^{3}$ Darabi, A. and Wygnanski, I., "Active Management of Naturally Separated Flow Over a Solid Surface Part 1: the Forced Reattachment Process," J. Fluid Mech., Vol. 510, 2004, pp. 105-129.

${ }^{4}$ Darabi, A. and Wygnanski, I., "Active Management of Naturally Separated Flow Over a Solid Surface Part 2: the Separation Process," J. Fluid Mech., Vol. 510, 2004, pp. 131-144.

${ }^{5}$ Amitay, M. and Glezer, A., "Flow Transients Induced on a 2-D Airfoil by Pulse-Modulated Actuation," Experiments in Fluids, Vol. 40, No. 2, 2006, pp. 329-331.

${ }^{6}$ Torres, G. E. and Mueller, T. J., "Low-Aspect-Ratio Wing Aerodynamics at Low Reynolds Numbers," AIAA J., Vol. 42(5), 2004, pp. 865-873.

${ }^{7}$ Williams, D. R., Tadmor, G., Colonius, T., Kerstens, W., Quach, V., and Buntain, S., "The Lift Response of a Stalled Wing to Pulsatile Disturbances," AIAA J., Vol. 47(12), 2009.

${ }^{8}$ Williams, D. R., Collins, J., Tadmor, G., and Colonius, T., "Control of a Semi-Circular Planform Wing in a "Gusting" Unsteady Free stream Flow I: Experimental Issues," AIAA Paper 2008-3976, 2008.

${ }^{9}$ Williams, D. R., Collins, J., Jankhot, C., Colonius, T., and Tadmor, G., "Control of Flow Structure on a Semi-Circular Planform Wing," AIAA Paper 2008-597, 2008.

${ }^{10}$ Williams, D. R., Quach, V., Kerstens, W., Buntain, S., Tadmor, G., Rowley, C., and Colonius, T., "Low-Reynolds Number Wing Response to an Oscillating Freestream with and without Feed Forward Control," AIAA Paper 2009-143, 2009.

${ }^{11}$ Colonius, T. and Williams, D. R., "Control of vortex shedding on two- and three-dimensional aerofoils," Phil. Trans. Royal Soc. A, Vol. 369, 2011, pp. 1525-1539.

${ }^{12}$ Raju, R., Mittal, R., and Cattafesta, L., "Dynamics of Airfoil Separation Control Using Zero Net Mass Forcing," AIAA J., Vol. 46(12), 2008, pp. 3103-3115.

${ }^{13}$ Brès, G. A., Williams, D. R., and Colonius, T., "Numerical Simulations of Natural and Actuated Flow over a 3D, Low-Aspect-Ratio Airfoil," AIAA Paper 2010-4713, 2010.

${ }^{14}$ Chen, H., Chen, S., and Matthaeus, W., "Recovery of the Navier-Stokes Equations Using a Lattice-gas Boltzmann Method," Phys. Rev. A, Vol. 45, 1992, pp. 5339-5342.

${ }^{15}$ Chen, H., Teixeira, C., and Molvig, K., "Digital Physics Approach to Computational Fluid Dynamics, Some Basic Theoretical Features," Intl. J. Mod. phys. C, Vol. 8(4), 1997, pp. 675.

${ }^{16}$ Chen, S. and Doolen, G., "Lattice Boltzmann Method for Fluid Flows," Ann. Rev. Fluid Mech., Vol. 30, 1998, pp. 329364.

${ }^{17}$ Shan, X., Yuan, X., and Chen, H., "Kinetic Theory Representation of Hydrodynamics: a way beyond the Navier-Stokes equation," J. Fluid Mech., Vol. 550, 2006, pp. 413-441. 
${ }^{18}$ Chen, H., Kandasamy, S., Orszag, S., Shock, R., Succi, S., and Yakhot, V., "Extended Boltzmann Kinetic Equation for Turbulent Flows," Science, Vol. 301, 2003, pp. 633-636.

${ }^{19}$ Chen, H., Orszag, S., Staroselsky, I., and Succi, S., "Expanded Analogy between Boltzmann Kinetic Theory of Fluid and Turbulence," J. Fluid Mech., Vol. 519, 2004, pp. 307-314.

${ }^{20}$ Li, Y., Shock, R., Shang, R., and Chen, H., "Numerical Study of Flow Past an Impulsively Started Cylinder by the Lattice-Boltzmann Method," J. Fluid Mech., Vol. 519, 2004, pp. 273-300.

${ }^{21}$ Fares, E., "Unsteady Flow Simulation of the Ahmed Reference Body using a Lattice Boltzmann Approach," Comput. Fluids, Vol. 35(8-9), 2006, pp. 940-950.

${ }^{22}$ Brès, G. A., "Flow and Noise Predictions for Tandem Cylinders in a Realistic Wind-Tunnel Configuration," AIAA Paper 2011-2824, 2011.

${ }^{23}$ Shock, R., Mallick, S., Chen, H., Yakhot, V., and Zhang, R., "Recent results on two-dimensional airfoils using a lattice Boltzmann-based algorithm," J. Aircraft, Vol. 39(3), 2002, pp. 434-439.

${ }^{24}$ Keating, A., Beedy, J., and Shock, R., "Lattice Boltzmann Simulations of the DLR-F4, DLR-F6 and Variants," AIAA Paper 2008-749, 2008.

${ }^{25}$ Kotapati, R., Keating, A., Kandasamy, S., Duncan, B., Shock, R., and Chen, H., "The Lattice-Boltzmann-VLES Method for Automotive Fluid Dynamics Simulation, a Review," SAE Paper 2009-26-057, 2009.

${ }^{26}$ Keating, A., Dethioux, P., Satti, R., Noelting, S., Louis, J., Van de Ven, T., and Vieito, R., "Computational Aeroacoustics Validation and Analysis of a Nose Landing Gear," AIAA Paper 2009-3154, 2009.

${ }^{27}$ Bearman, P. W., "On vortex street wakes," J. Fluid Mech., Vol. 28, 1967, pp. 625641.

${ }^{28}$ Greenblatt, D. and Wygnanski, I. J., "The control of flow separation by periodic excitation," Prog. Aerosp. Sci., Vol. 28, 2000, pp. 73-131.

${ }^{29}$ Brzozowski, D. and Glezer, A., "Transient Separation Control Using Pulse-Combustion Actuation," AIAA Paper 2006$3024,2006$.

${ }^{30}$ Woo, G., Crittenden, T., and Glezer, A., "Transitory control of a pitching airfoil using pulse combustion actuation," AIAA paper 2008-4324, 2008.

${ }^{31}$ Siauw, W. L., Bonnet, J.-P., Tensi, J., Cordier, L., Noack, B. R., and Cattafesta, L., "Transient dynamics of the flow around a NACA 0015 airfoil using fluidic vortex generators," Int. J. Heat and Fluid Flow, Vol. 31, 2010, pp. 450-459. 